\title{
Generation of Third and Higher-Order Intermodulation Products in MEMS Capacitors, and their Effects
}

\author{
David Girbau $^{1}$, Nerea Otegi ${ }^{2}$, Lluís Pradell ${ }^{1}$, Antonio Lázaro ${ }^{3}$ \\ ${ }^{1}$ Universitat Politècnica de Catalunya, Signal Theory and Communications Department, 1-3 Jordi Girona St., 08034 \\ Barcelona, Spain. Tel. +34-93.401.68.34 \\ ${ }^{2}$ University of the Basque Country, Electricity and Electronics Department, 48080 Bilbao, Spain. \\ ${ }^{3}$ Universitat Rovira i Virgili, Electronics, Electrical and Automatics Engineering Department, 43007 Tarragona, Spain.
}

\begin{abstract}
In this work, the Intermodulation Distortion (IMD) produced by RF MEMS capacitors is studied. It is demonstrated that due to their non-linear behaviour, parallel-plate MEMS capacitors generate $5^{\text {th }}$ order tones along with $3^{\text {rd }}$ order IMD products. It is shown that MEMS linear models, restricted to small displacements of the MEMS membrane, neglect $5^{\text {th }}$ order distortion while underestimate $3^{\text {rd }}$ order tones, when high voltages are considered. A numerical non-linear model for simulating intermodulation is presented and validated by means of measurements for the general 2-tone case. Generation of $3^{\text {rd }}$ and $5^{\text {th }}$ IMD products is also demonstrated in MEMS driven by digitally-modulated communication signals. A measurement system is proposed in order to characterize IMD generation in RF MEMS capacitors excited by two RF tones as well as by digitally-modulated signals (QPSK).
\end{abstract}

\section{INTRODUCTION}

One of the main characteristics of RF MEMS is to generate lower intermodulation distortion (IMD) than their semiconductor equivalents. However, under some excitation conditions, IMD can be important in RF MEMS. Although IMD can frequently be neglected in switches [1], it becomes significant in variable capacitors, due to their higher nominal capacity (membrane in the up state) and non-linear behaviour. This work demonstrates the generation of $5^{\text {th }}$ and higher order intermodulation products in MEMS capacitors, showing that distortion is not limited to $3^{\text {rd }}$ order. These high-order products are a direct consequence of the nonlinear response of the membrane to the applied voltage. In fact, it is shown that, due to its non-linear behaviour, the membrane mean displacement increases with respect to the one produced by DC-bias and self-actuation, and so all IMD products increase in turn.

An analytical model for $3^{\text {rd }}$ order IMD in MEMS switches has already been presented [1], along with measurements of $3^{\text {rd }}$ order IMD. However, in [1] two assumptions are made, small displacements (linear approximations are allowed) and bridge high-impedance, that are not generally fulfilled in two parallel-plate capacitors. This is because MEMS variable capacitor membranes can present displacements up to $33 \%$ of the initial gap (tuning range), and their higher nominal capacity corresponds to an impedance that can not be considered an open circuit. Another model, based on Volterra-Series, is presented in [2], which obtains simulated $3^{\text {rd }}$ order IMD in MEMS capacitors.

The objectives of this work are: first, to provide a physical explanation of the origin of IMD in MEMS devices based on a mobile membrane; it is shown that not only $3^{\text {rd }}$ order IMD is produced but also higher orders, as a direct consequence of the membrane non-linear behaviour. The second is to observe the effects of IMD in MEMS capacitors, by providing numerical simulations for the worst case of IMD in MEMS, i.e., when the difference-frequency of the two input RF tones (for the general case of a 2-tone test) is within the device mechanical bandwidth. Measurements of IMD up to $5^{\text {th }}$ order are also presented and compared to simulations. A second phenomenon due to the two RF tones at the capacitor is a time-varying capacity, which can be significant when the membrane is positioned in the strongly non-linear regions of the tuning range. Finally, the effect of MEMS IMD on digitally-modulated communication signals is studied. A measurement set-up to measure IMD for 2-tone test as well as for digital modulations is proposed.

\section{Displacement of the Mobile Membrane Due to THE PRESENCE of Two RF TONES AND A Bias Voltage}

The dynamics of motion of a suspended membrane of an electrostatically-actuated two parallel-plate capacitor can be approximated by the 1-D non-linear differential equation of a mass-spring-damper system:

$$
m \frac{d^{2} x}{d t^{2}}+b \frac{d x}{d t}+k x=\frac{1}{2} \frac{\varepsilon A V^{2}}{(d-x)^{2}}
$$

where $x$ is the displacement, $d$ the initial gap, $A$ the electrodes area, $V$ the applied voltage and $\varepsilon$ the air permittivity; $m, b$ and $k$ are the mass, damping coefficient and total suspension stiffness constant of the mobile plate, respectively. Assuming a signal composed of two $\mathrm{RF}$ tones and DC-bias at the capacitor:

$$
V=V_{\text {bias }}+V_{1} \sin \omega_{1} t+V_{2} \sin \omega_{2} t
$$

The square of voltage $V$ in (1) can be approximated as:

$V^{2} \approx V_{\text {bias }}^{2}+\frac{V_{1}^{2}}{2}+\frac{V_{2}^{2}}{2}+V_{1} V_{2} \cos \left(\omega_{1}-\omega_{2}\right) t=V_{D C}+V_{t} \cos (\omega t)$

where high-frequency terms have been omitted, since, due to the low-pass behaviour of (1), they will have no influence on the position of the membrane. It must be stressed that $\mathrm{V}_{1}$ and $\mathrm{V}_{2}$ are the total voltages at the capacitor (which must be calculated in each circuit configuration), not the amplitudes of the incident waves $\left(\mathrm{V}_{01}{ }^{+}, \mathrm{V}_{02}{ }^{+}\right)$. In order to study the origin of IMD in MEMS, a difference frequency $\omega<<\omega_{0}\left(\omega_{0}\right.$, mechanical resonance) is assumed, being this the worst case for IMD generation, due to the low pass character of equation (1). 
With this assumption, the membrane response $x(t)$ can be calculated as a series of successive, instantaneous, independent solutions obtained at time instants $t_{j}$, at which the squared voltage (from (3)) is defined as $V^{2}\left(t_{j}\right)=V_{D C}+V_{t} \cos \left(\omega t_{j}\right)$. Substituting it into (1), the instantaneous, steady displacement $x\left(t_{j}\right)$ is:

$$
k x\left(t_{j}\right)=\frac{1}{2} \frac{\varepsilon A V^{2}}{\left(d-x\left(t_{j}\right)\right)^{2}}=\frac{1}{2} \frac{\varepsilon A\left(V_{D C}+V_{t} \cos \left(\omega t_{j}\right)\right)}{\left(d-x\left(t_{j}\right)\right)^{2}}
$$

If we assume that the membrane is describing a sinusoidal movement at $\omega$, superposed to a DC displacement due to bias voltage and self-actuation $x_{D C}=x\left(V_{D C}\right)$ [1], the displacement could be expressed as:

$$
k x_{\text {linear }}(t) \approx \frac{1}{2} \frac{\varepsilon A V_{t} \cos (\omega t)}{\left(d-x_{D C}\right)^{2}}, \quad k x_{D C}=\frac{1}{2} \frac{\varepsilon A V_{D C}}{d^{2}}
$$

where only $3^{\text {rd }}$ order IMD products are considered. Indeed, this linear behaviour is observed for low capacitor voltages, and also for a capacitive switch with low self-actuation (low RF power) in its ON state, but it is not a good model for the displacement generated by two RF tones in a MEMS capacitor, as it can be concluded from Fig.1, which illustrates the generation of displacement higher order harmonics.

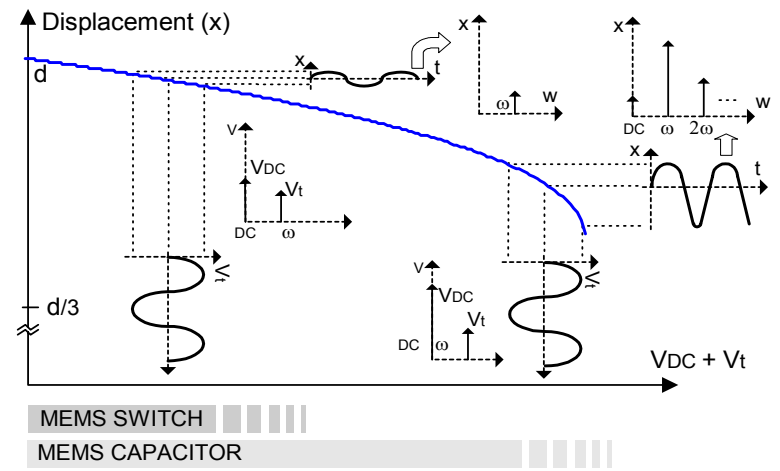

Fig.1. Displacement of the mobile membrane of a MEMS capacitor due to the two RF tones and a bias voltage.

Due to the non-linear nature of (4), and as can be qualitatively appreciated in Fig.1, the sinusoidal movement supposed in (5) degenerates as the system is driven by high input voltages, produced by either bias voltage or RF power. Considering harmonics up to the $3^{\text {rd }}$ order, the following expression for the displacement (non-linear model) is proposed:

$$
x(t) \approx \bar{x}+\sum_{k=1}^{3} A_{k} \cos \left(k \omega t+\delta_{k}\right)
$$

The $A_{k}$ amplitudes can be directly derived from a Fourier Transform of $x(t)$ obtained in (4). A comparison of the linear approximation given by (5) to the non-linear solution of (4) and to the model of (6) is shown in Fig.2 for two cases. Fig.2.a shows the case of low voltages at the capacitor $\left(\mathrm{P}_{\mathrm{RF}}=10 \mathrm{dBm}\right.$ and $\left.\mathrm{V}_{\text {bias }}=0\right)$, where a good agreement between the displacements calculated from (4) , (5) and (6) is observed, as expected. Fig.2.b shows the case of high voltages at the capacitor $\left(\mathrm{P}_{\mathrm{RF}}=13.9 \mathrm{dBm}\right.$ and $\mathrm{V}_{\text {bias }}=0$ ), where higher harmonics of $\omega$ become significant, causing $5^{\text {th }}$ (and higher order) IMD products to appear and (eventually) be measurable, as will be demonstrated through measurements of $5^{\text {th }}$ order distortion provided in section IV. In this case, the linear approximation (5) is no longer valid: the main sinusoid at $\omega$ itself is undervalued by the linear model and, as a consequence, $3^{\text {rd }}$ order intermodulation would also be underestimated. Moreover, in (5), the term at $2 \omega$ (the one which generates $5^{\text {th }}$ order intermodulation) is neglected.

In addition to this phenomenon, as can be observed in Fig.2.b, the DC position of the membrane is also affected by the non-linear behaviour of the system. The mean membrane position $\bar{x}=\overline{x(t)}$ is not only determined by $V_{D C}$ (bias + self-actuation), but an additional contribution to the displacement emerges from non-linear terms in equation (4).

An important remark is that these two effects are enhanced for membrane positions in the highly nonlinear regions of the tuning range (near pull-in), a situation that may happen in electrostatically-actuated two parallel-plate varactors, where membrane positioning may go across all the tuning range.
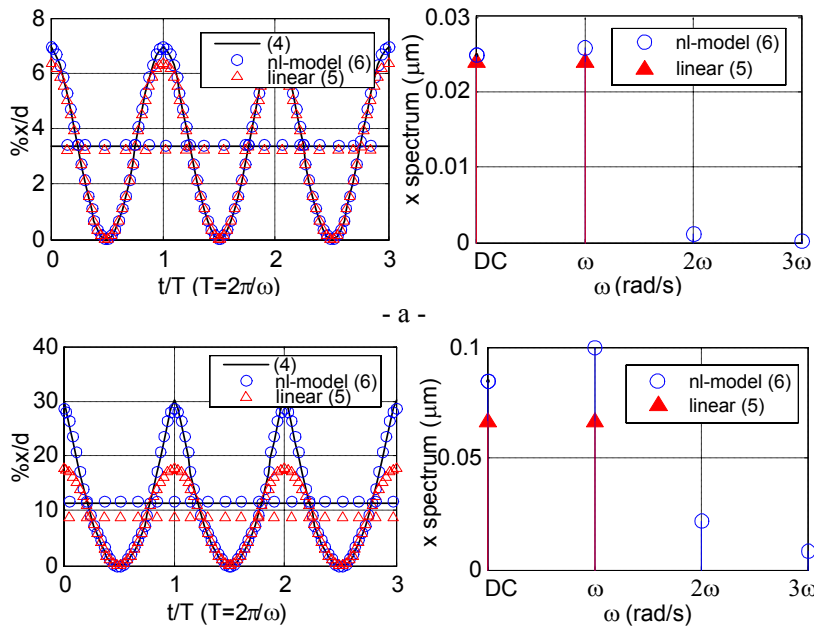

$-b$ -

Fig. 2. Comparison of time waveforms and spectrum obtained from the linear approximation (5), the rigorous solution of (4) and the displacement from (6), for the case of low voltages at the capacitor (a) and high voltages (b). Capacitor parameters: see section IV.

\section{INTERMODULATION GENERATION}

Since the capacity depends on the instantaneous gap, the reflection coefficient is a function of the displacement. The capacitor model is the one proposed in Fig.4, where a loss resistance $R$ and a parasitic capacity $C_{P}$ have been added for generalisation purposes. The reflection coefficient phase at a frequency $\omega_{i}$, is then given by:

$$
\Phi_{i}(t)=\angle \Gamma=a \tan \left(\frac{2 Z_{0} \omega_{i}\left(C_{p}+\frac{\varepsilon A}{(d-x(t))}\right) R^{2}}{R^{2}-Z_{0}^{2}-\left(Z_{0} \omega_{i} R\right)^{2}\left(C_{p}+\frac{\varepsilon A}{(d-x(t))}\right)^{2}}\right)
$$

Expanding (7) in a Taylor series of $(x(t)-\bar{x})$ and substituting (6), the phase steady-state and harmonics are obtained:

$$
\begin{aligned}
& \Phi_{i}(t) \approx \Phi_{i}(\bar{x})+\left.\frac{d \Phi_{i}}{d x}\right|_{x=\bar{x}}(x(t)-\bar{x})+\left.\frac{1}{2} \frac{d^{2} \Phi_{i}}{d x^{2}}\right|_{x=\bar{x}}(x(t)-\bar{x})^{2} \\
& \approx \Phi_{i}(\bar{x})+\left.\frac{d \Phi_{i}}{d x}\right|_{x=\bar{x}}\left(\sum_{k=1}^{3} A_{k} \cos \left(k \omega t+\delta_{k}\right)\right)+\left.\frac{1}{2} \frac{d^{2} \Phi_{i}}{d x^{2}}\right|_{x=\bar{x}}\left(\sum_{k=1}^{3} A_{k} \cos \left(k \omega t+\delta_{k}\right)\right)^{2}
\end{aligned}
$$


A quadratic approach is sufficient to characterize the temporal behaviour of $\Phi_{\mathrm{i}}(\mathrm{t})$, as can be seen in Fig.3.a, where the reflection coefficient phase is plotted vs. time for the near pull-in case of Fig.2.b. The non-linear terms of the phase vs. displacement have a small contribution to the total IMD. The reflected voltage $V_{r}$ at the MEMS capacitor is:

$$
V_{r}=|\Gamma| V_{01}^{+} \sin \left(\omega_{1} t+\Phi_{1}(t)\right)+|\Gamma| V_{02}^{+} \sin \left(\omega_{2} t+\Phi_{2}(t)\right)
$$

where $|\Gamma|$ is the reflection coefficient magnitude. It is clear from (8) and (9) that the reflected signal presents a periodical phase modulation, caused by the displacement $x(t)$, which generates the IMD products. Fig.3.b shows the reflected power spectrum $\mathrm{P}^{-}$, which is derived from (9), for the case of high RF power. High order IMD products, not taken into account by a linear approximation (5), are clearly observed now, as well as an underestimation of the $3^{\text {rd }}$ order products.
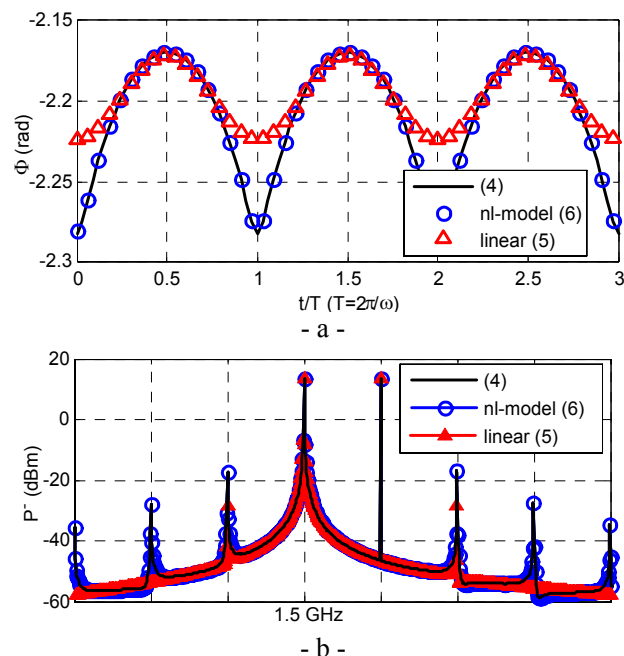

Fig.3. Reflection coefficient phase (a) and IMD power spectrum (b) for the case of $\mathrm{P}_{\mathrm{RF}}=13.9 \mathrm{dBm}$ and no bias voltage. Capacitor parameters: see section IV.

\section{Model VALIDATION AND MEASUREMENTS}

Fig.4 shows the measurement system proposed to characterize IMD produced by MEMS capacitors excited with 2-tone and digitally-modulated signals, based on the one presented in [3]. An on-wafer MEMS capacitor is used to validate the proposed non-linear model, whose effective parameters are: $K=35 \mathrm{~N} / \mathrm{m}, C=1.43 \mathrm{pF}, C_{P}=2.57$ $\mathrm{pF}, R=361 \Omega, d=0.75 \mu \mathrm{m}$.

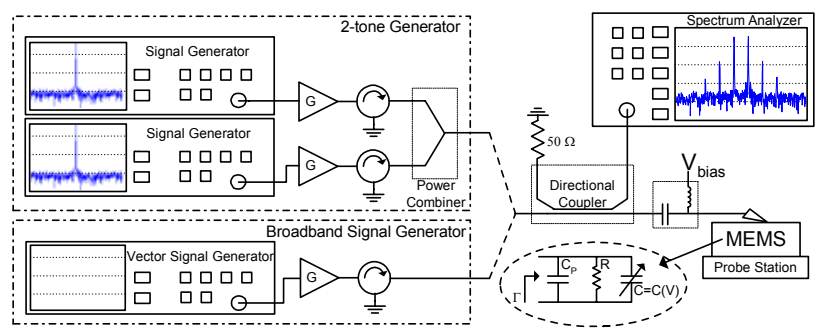

Fig.4. IMD measurement system for MEMS capacitors.

Fig.5.a. shows the measured reflected signal at the capacitor for an injected $\mathrm{RF}$ power $\mathrm{P}_{\mathrm{RF}}=11 \mathrm{dBm}$ and $\mathrm{a}$ frequency separation between RF tones of $100 \mathrm{~Hz}$ (well within the mechanical bandwidth, $\omega_{0}$ ), where $3^{\text {rd }}$ and $5^{\text {th }}$ order IMD products are noticeable. Their measured frequency dependence is plotted in Fig.5.b and compared to the IMD predicted using both, the linear and nonlinear displacement approximations for several frequency points $\omega<\omega_{0}$. It is observed that IMD is significant for frequencies $\omega$ within the mechanical bandwidth. Therefore, the calculation of IMD in this range as a worst case, is justified.
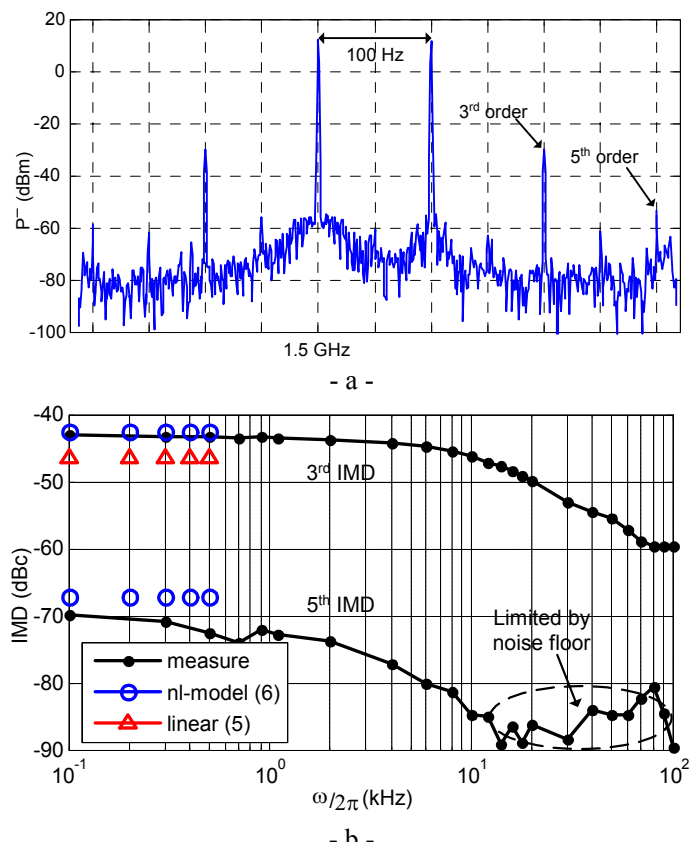

Fig.5. Spectrum of the reflected signal at the capacitor (a) and frequency-dependence of $3^{\text {rd }}$ and $5^{\text {th }}$ order intermodulation products, compared to the linear approximation and to the proposed non-linear model for the case of $\omega<\omega_{0}$ (b). $\mathrm{P}_{\mathrm{RF}}=11$ $\mathrm{dBm}$.

Fig. 6 shows intermodulation measurement results as a function of RF power, compared to both, linear approximation (5) and the proposed non-linear numerical model.

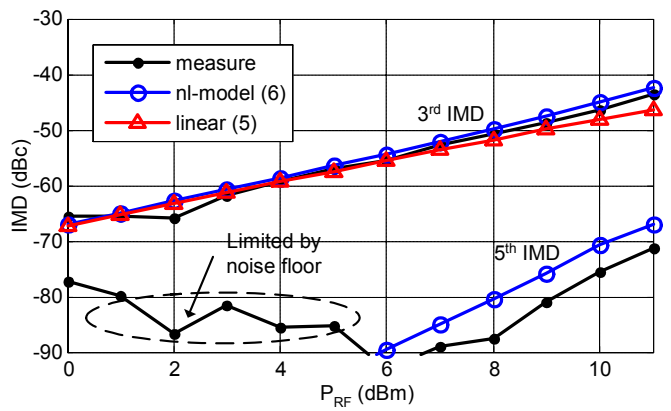

Fig.6. $3^{\text {rd }}$ and $5^{\text {th }}$ order intermodulation measurement results, compared to the linear prediction and to the numerical nonlinear model. $\omega=2 . \pi .1 \mathrm{kHz}$.

Concerning the $3^{\text {rd }}$ order product, observe that, for high power excitation, taking into account higher harmonics (as it is considered in (6)) is important for an accurate $3^{\text {rd }}$ order distortion prediction. Furthermore, as power increases, a significant $5^{\text {th }}$ order distortion emerges, neglected by the linear approximation. Nevertheless, the presented non-linear model predicts a $5^{\text {th }}$ order IMD higher than measured, which can be attributed to the fact that the mobile membrane does not move as a rigid block and therefore, the displacement sinusoid is not as deformed as predicted (see Fig.2). 
A second, simultaneous effect of the two RF tones at the capacitor is a time-varying capacity, at a frequency $\omega$. Fig.7 shows this effect for $3 \mathrm{RF}$ powers $\mathrm{P}_{\mathrm{RF}}$ at the capacitor. It can be observed that this variation can be significant as the membrane is placed in the strongly nonlinear regions.
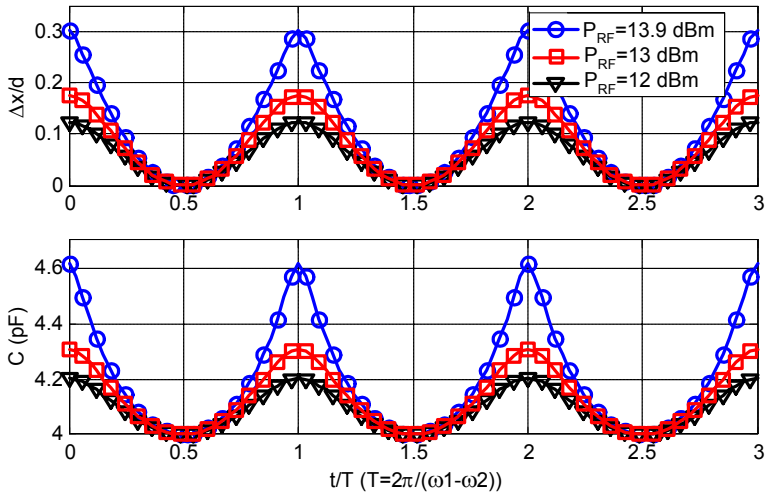

Fig.7. Time-dependent capacity due to the two RF tones at the capacitor. $\omega=\omega_{1}-\omega_{2}=2 . \pi .100 \mathrm{~Hz}\left(<<\omega_{0}\right)$.

If the two tones at the capacitor are replaced with a digitally-modulated communication signal (QPSK), both, $3^{\text {rd }}$ and $5^{\text {th }}$ order products come up, which worsen the ACPR. This situation is illustrated in Fig. 8, showing a measurement of ACPR as a function of symbol rate $R_{S}$. An important conclusion can be drawn: those applications using symbol rates comparable to or lower than the mechanical bandwidth of the MEMS device $\left(\mathrm{R}_{\mathrm{S}}<\omega_{\mathrm{o}} / 2 \pi\right)$ will suffer from an ACPR degradation, while intermodulation distortion will not be an important issue when higher symbol rates are considered.

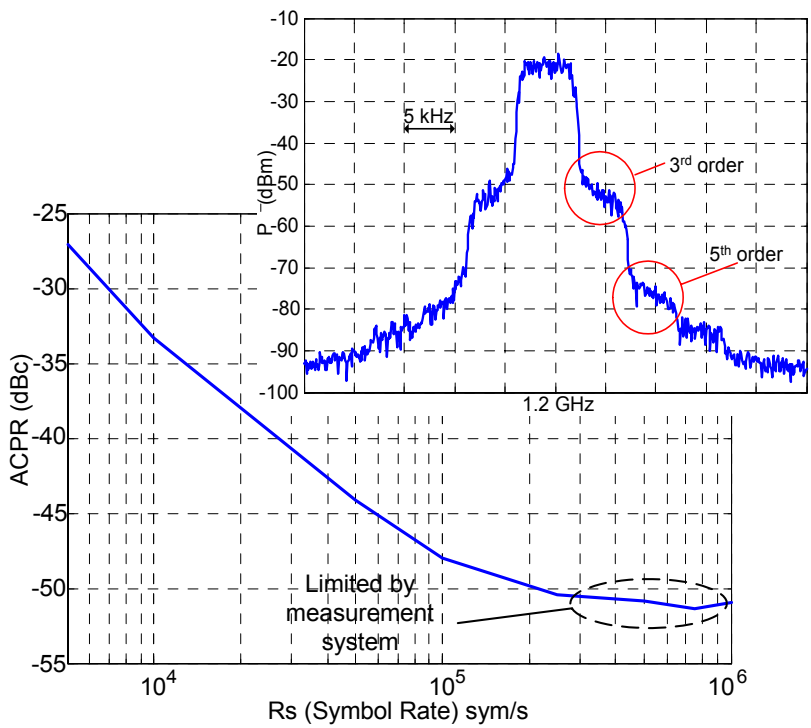

Fig. 8. ACPR versus symbol rate $\mathrm{R}_{\mathrm{S}}$, for an input QPSK signal at $1.2 \mathrm{GHz}, \mathrm{P}_{\mathrm{RF}}=+17 \mathrm{dBm}$ and roll-off factor=0.3. Spectrum of the reflected signal at the capacitor, with $\mathrm{R}_{\mathrm{S}}=5 \mathrm{ks} / \mathrm{s}$.

The dependence of ACPR on the RF power at the capacitor is shown in Fig.9, along with the degradation of the spectrum of the reflected signal.
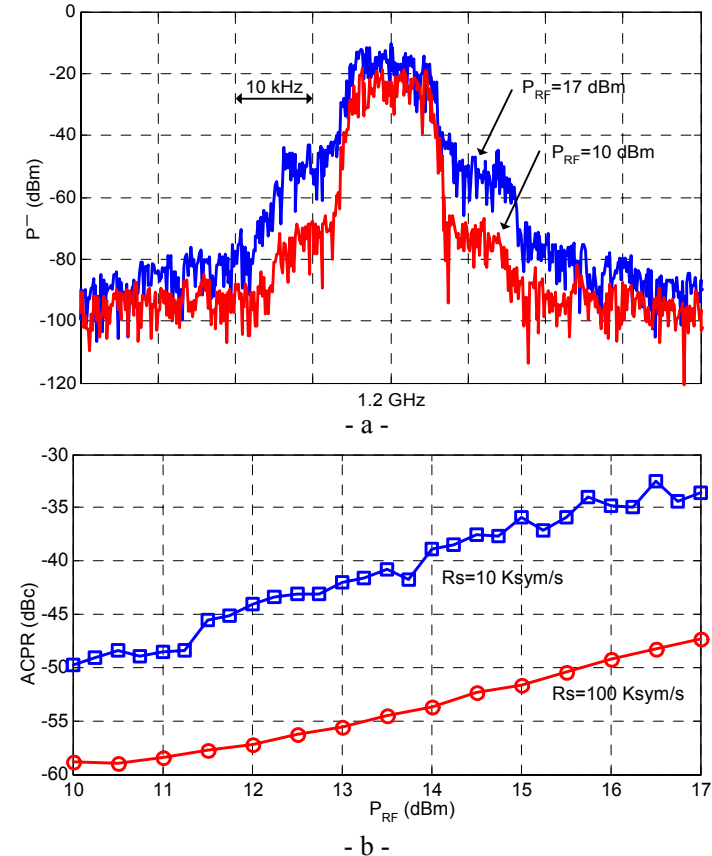

Fig. 9. Spectrum of the reflected signal with symbol rate $\mathrm{R}_{\mathrm{S}}=10 \mathrm{ksym} / \mathrm{s}$ (a) and dependence of ACPR on the RF power at the capacitor (b) for a QPSK-modulated signal. Rolloff $=0.3$.

\section{CONCLUSION}

This paper has presented a numerical non-linear model for predicting intermodulation distortion (IMD) in MEMS capacitors for the general case of a two-tone test, and validated by means of measurements. It is demonstrated that using non-linear expressions is a requirement to obtain an accurate prediction of third order IMD products. It is also shown that fifth order IMD products, neglected by linear approximations, present significant and measurable values. A second effect due to the two RF tones has been presented, namely a timevarying capacity. Third and fifth order distortions have also been measured in MEMS excited with digitallymodulated signals, showing a dependence on symbol rate: ACPR worsens for symbol rates comparable to or lower than the mechanical resonance frequency of the device, while for higher symbol rates, the ACPR is not affected by the MEMS non linearity. The ACPR also shows a degradation with increasing RF power.

\section{ACKNOWLEDGEMENT}

This work has been financed by the Spanish Government Project ESP2004-07067-C03-03 (MCYT).

\section{REFERENCES}

[1] L. Dussopt, G.M.Rebeiz, "Intermodulation Distortion and Power Handling in RF MEMS Switches, Varactors, and Tunable Filters", IEEE Trans. Microwave Theory and Tech., Vol.51, No4, Apr. 2003.

[2] M. Innocent, P. Wambacq, S. Donnay, H. A. C. Tilmans, W. Sansen, H. De Man, "An analytic Volterra-Series-Based Model for a MEMS Variable Capacitor”, IEEE Trans. Computer-Aided Design of Int. Circuits and Systems, Vol.22, No2, Feb. 2003.

[3] D.Girbau, A.Lázaro, L.Pradell, "Characterization of dynamics and power handling of RF MEMS using vector measurement techniques", IEEE Trans. on Microwave Theory and Techniques, Vol. 52, $N^{o} 11$, pp. 2627-2633, Nov. 2004. 